\title{
Characterization of Structure and Magnetic Properties of Warm Compacted Ni-Fe-Mo Soft Magnetic Alloy
}

\author{
P. SlovenskÝ $\bar{Y}^{a, *}$, P. Kollár ${ }^{a}$, M. JakubČIn ${ }^{a}$, J. FÜZer ${ }^{a}$, \\ D. OlekšÁKOvá ${ }^{b}$, M. FÁBerovác and R. Bureš ${ }^{c}$ \\ ${ }^{a}$ Institute of Physics, Faculty of Science, P.J. Šafárik University, Park Angelinum 9, 04154 Košice, Slovakia \\ ${ }^{b}$ Institute of Manufacturing Management, Faculty of Manufacturing Technologies, \\ Technical University of Košice, Bayerova 1, 08001 Prešov, Slovakia \\ ${ }^{c}$ Institute of Materials Research, Slovak Academy of Sciences, Watsonova 47, 04001 Košice, Slovakia \\ Surface morphology and basic soft magnetic properties were investigated on Ni80Fe15Mo5 compacted pow- \\ der alloys. The powder was prepared by grinding of the Ni80Fe15Mo5 sheet, followed by milling and surface \\ smoothing. The powder was warm compacted to get ring shaped compacts, which were then annealed. The effects of \\ surface smoothing on the microstructure and magnetic properties were examined. The relative initial permeability, \\ coercivity and specific electrical resistance measurements were conducted on both un-annealed and annealed \\ samples. The initial relative permeability (at $100 \mathrm{~Hz}$ ) has increased its value from 120 for non-smoothed sample \\ to 200 after smoothing, and to 3300 after annealing. For annealed non-smoothed sample the initial relative per- \\ meability was 1500 . The lowest coercivity was measured after powder-smoothing and annealing, where the sample \\ exhibited coercivity of $4.8 \mathrm{~A} / \mathrm{m}$. The values of specific electrical resistivity were relatively stable, keeping the value \\ around $1 \times 10^{-6} \Omega \mathrm{m}$
}

DOI: 10.12693/APhysPolA.137.876

PACS/topics: soft magnetic materials, powder compacted materials, powder metallurgy, magnetic measurements

\section{Introduction}

NiFe (permalloy) and NiFeMo (supermalloy) alloys are known for their high permeability, low coercivity and near-to-zero magnetostriction. Further, they are widely used in magnetic cores and magnetic shielding of electrical components, such as transformers or electric sensors in the form of thin sheets [1]. For the exceptional soft magnetic properties, alloys can be used as a base for the production of soft magnetic powder compacted materials for DC and low frequency applications. Since generated alloys are generated by conventional powder metallurgy, they give an opportunity to produce components of different and precise shapes with minimal waste [2-5]. There are many factors that can affect the magnetic properties obtained, either during the metal powder preparation or during the compaction. In fact, every step in the material preparation process affects in some way (positively or negatively) the final magnetic properties, and more or less significantly. For example, compacted powder material after suitable heat treatment can achieve soft magnetic properties comparable to the material prepared by conventional methods (as casting) [6, 7]. The same applies to higher compact density, which can be reached by compacting at high pressures. This, however, leads to accumulation of inner defects that hinder the displacement of domain walls

\footnotetext{
* corresponding author; e-mail: peter.slovensky@student.upjs.sk
}

during magnetization, resulting in increased coercivity and hysteresis losses. That is why the defects must be removed by heat treatment [8].

The effect of mechanical treating on the powder particle shape and the resulting improvement in magnetic properties has been already described [9]. The aim of this work was to investigate the effects of surface smoothing on the surface morphology of powdered Ni80Fe15Mo5 alloy, the resulting impact on magnetic and microstructural properties of warm compacted material

\section{Experimental}

The powder particles were prepared by milling of small chips (1-5 mm, by Retch PM100 planetary ball mill in steel vial with steel balls for $3 \mathrm{~h}$, BPR 9:1, rpm 300), which were obtained by rotary grinder from NiFeMo sheets (0.6 mm thick). NiFeMo powder (80 wt.\% Ni, 15 wt. $\% \mathrm{Fe}$, and 5 wt. $\% \mathrm{Mo})$ was sieved to $100-300 \mu \mathrm{m}$ particle size. The mechanical treatment procedure was performed in order to smooth the edges of surface irregularities of milled powder (detailly described in [9]). The ring-shaped samples were produced by conventional powder metallurgy, where inner diameter is $18 \mathrm{~mm}$, and outer diameter is $24 \mathrm{~mm}$. The powder was warm compacted under vacuum at pressure of $700 \mathrm{MPa}$ and temperature of $410^{\circ} \mathrm{C}$ for $5 \mathrm{~min}$. The annealing procedure followed after the compaction and was performed for $10 \mathrm{~h}$ in hydrogen atmosphere at the temperature of $1100^{\circ} \mathrm{C}$ (heating rate $10^{\circ} \mathrm{C} / \mathrm{min}$, hold-up period $6 \mathrm{~h}$, and cooling rate $10^{\circ} \mathrm{C} / \mathrm{min}$ ). For further analysis, four samples were selected: sample A - prepared from the irregular particles; sample B - similarly 
as sample A, but compaction was followed by annealing; sample $\mathrm{C}$ - prepared from surface smoothed particles; and sample D - similarly as sample C, but compaction was followed by annealing. Closer details of sample preparation were described in [9].

For the metallographic analysis, the samples were embedded in a conductive resin. Then they grinded and etched by Marble's Reagent for $45 \mathrm{~s}$. The surface morphology was visualized by scanning electron microscope (JEOL JSM 7000F). The density of samples was calculated as fraction mass to sample volume. Magnetic measurements were studied by means of the coercivity measurements (Foester Koerzimat 1.097 HCJ), measurements of the total energy losses at the maximum magnetic induction $B_{\max }=0.2 \mathrm{~T}$ in frequency range from DC to $100 \mathrm{~Hz}$ (AMH-1K-S hysteresisgraph), and the complex permeability measurements (impedance analyzer HP4194A with frequency range from $100 \mathrm{~Hz}$ to $40 \mathrm{MHz})[9,10]$.

\section{Surface morphology}

The most visible change after surface smoothing is the change in the shape of the powder particle [9]. Moreover, the surface smoothing also affects the surface roughness of the powders which is clearly visible at larger magnification. The surface of the non-treated sample is rough, covered with many microscopic protrusions. In turn, the surface of the treated sample is much smoother, with a significantly smaller appearance of protrusions (Fig. 1).

It is known that the shape of the particles significantly affects the technological properties, namely apparent density, fluidity, compressibility and porosity. In addition, numerous protrusions on the surface cause that the powder stuck together, thus reducing porosity and increasing the strength of a compact $[11,12]$.

\section{Magnetic properties}

To prove the positive influence of the particle surface smoothing on magnetic properties of prepared compacts we measured the real part of complex permeability at the frequency of $100 \mathrm{~Hz}$, the frequency dependence of the total energy losses, and the coercivity. The highest value of the real part of the relative initial permeability of 3300 was detected for sample D (with surface smoothed particles and annealed), while for sample B it was only 1500. Similarly for non-annealed samples, the permeability has increased its value from 120 for nonsmoothed sample, to 200 after smoothing. The positive effect of the surface smoothing is proven by coercivity measurements. The lowest value of the coercivity exhibits again sample $\mathrm{D}(4.8 \mathrm{~A} / \mathrm{m})$ due to positive influence of the surface smoothing procedure, when compared the coercivity $(24.4 \mathrm{~A} / \mathrm{m})$ for sample $\mathrm{B}$, prepared by the same way, without surface smoothing. The improvement of the soft magnetic properties can be explained
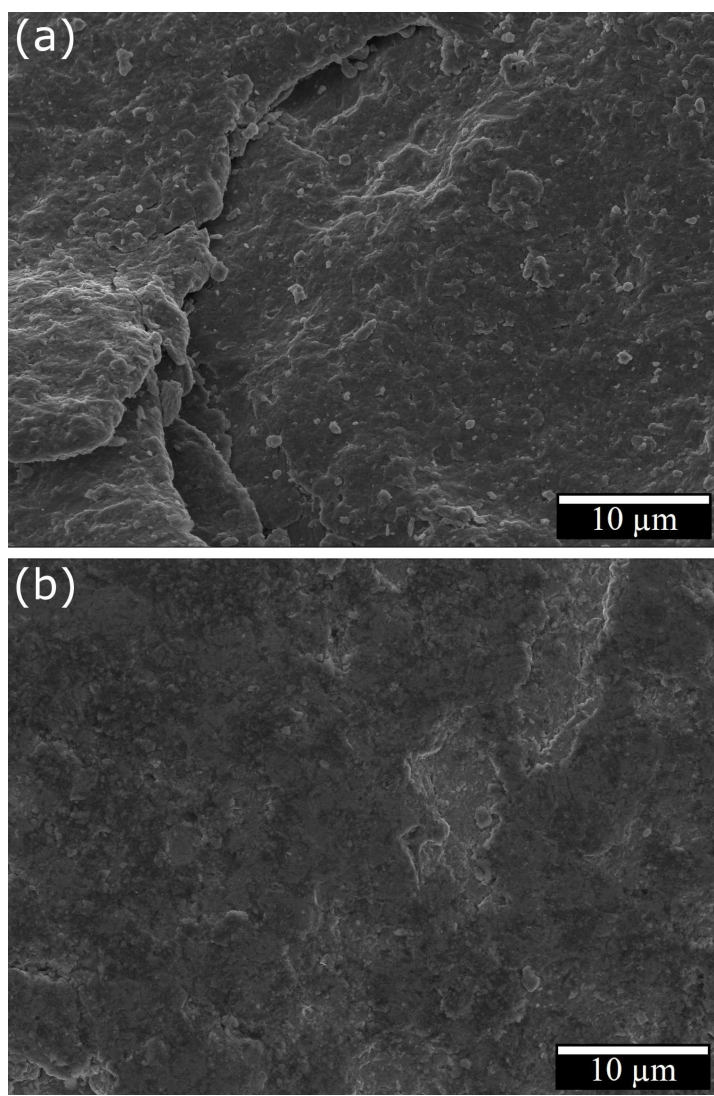

Fig. 1. (a) Surface of the irregular particle, and (b) particle after surface smoothing (magnification 2500×).

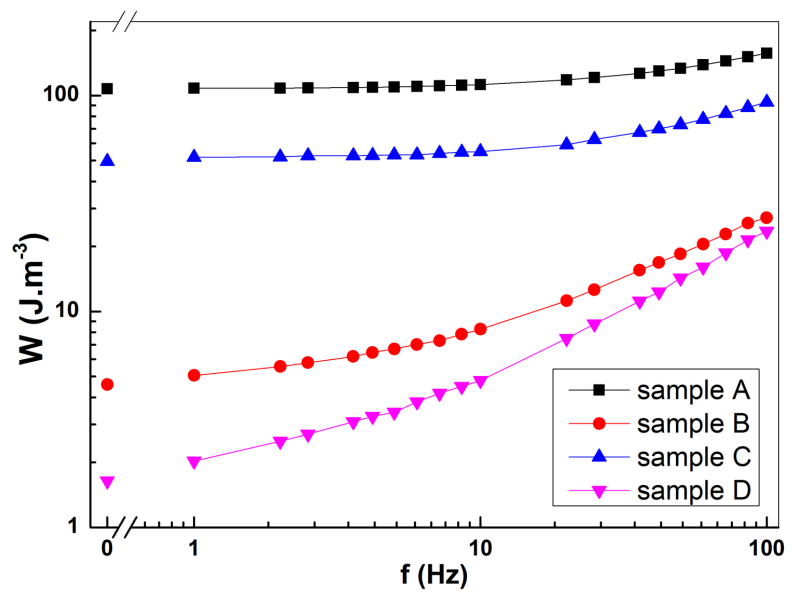

Fig. 2. Frequency dependence of the total energy losses at maximum magnetic induction $B_{\max }=0.2 \mathrm{~T}$ of the studied samples.

by reducing of significant amount of surface, hinders for domain wall displacement. Similarly, the decrease of total energy losses can be observed as well in Fig. 2 due to surface smoothing. The lowest value of total losses exhibits sample D with significant decrease of hysteresis loss (DC measurement). 


\section{Conclusion}

By investigating of the morphology of $\mathrm{Ni}_{80} \mathrm{Fe}_{15} \mathrm{Mo}_{5}$ powder particles surface used for preparation of soft magnetic compacts, we detected the origin of negative influence of the particles morphology on soft magnetic properties. The particles prepared by milling of small chips obtained by rotary grinder exhibit irregular protrusions on their surfaces. It is expected that smoother surfaces of powder elements contribute to more homogeneous pressure during compaction, what is positive for creation of soft magnetic bulk material. The protrusions on nonsmoothed surfaces of the powder elements generate areas with increased stresses. On the other hand, removing of the surface protrusions leads to more-spherical shape of the powder elements, what is responsible for lower density of prepared compacts, due to unfilled spaces between particles. Despite the lower density of resulting compacts, they show better soft magnetic properties. The best magnetic properties exhibits the sample prepared from particles with smoothed surfaces, consequently annealed at $1100^{\circ} \mathrm{C}$ for $10 \mathrm{~h}$ in hydrogen atmosphere: the coercivity was $4.8 \mathrm{~A} / \mathrm{m}$, and initial relative permeability at $100 \mathrm{~Hz}$ was 3500 . The significant improvement of magnetic properties of this sample is explained by removing of the surface protrusions acting as hinders for domain wall displacements.

\section{Acknowledgments}

This work was realized within the frame of the project "MACOMA" financed by Slovak Research and Development Agency under the contract APVV-15-0115; the Scientific Grant Agency of the Ministry of Education, Science, Research and Sport of the Slovak Republic and the Slovak Academy of Sciences (VEGA 1/0143/20 and VEGA 1/0301/20), the Internal Scientific Grant of the Faculty of Science, P.J. Šafárik University in Košice (vvgs-pf-2019-1038), and by the Development
Operational Programme Research and Innovation for the project" New unconventional magnetic materials for applications", ITMS: 313011T544, co-funded by the European Regional Development Fund (ERDF). This work was also realized under the contract of Cultural and Educational Grant Agency of the Ministry of Education, Science, Research and Sport of the Slovak Republic and the Slovak Academy of Sciences KEGA 002TUKE-4/2019.

\section{References}

[1] J. Ma, M. Qin, X. Wang, L. Zhang, L. Tian, X. Zhang, X. Li, X. Qu, Powder Technol. 253, 158 (2014).

[2] J. Füzer, P. Kollár, D. Olekšáková, S. Roth, J. Alloys Compd. 498, 557 (2009).

[3] K.J. Sunday, M.L. Taheri, Met. Powder Rep. 72, 425 (2017).

[4] D. Olekšáková, J. Füzer, P. Kollár, S. Roth, J. Magn. Magn. Mater. 333, 18 (2013).

[5] H. Shokrollahi, K. Janghorban, J. Mater. Process. Technol. 189, 1 (2007).

[6] H. Shokrollahi, K. Janghorban, Mater. Sci. Eng. B 134, 41 (2006).

[7] W. Ding, L. Jiang, Y. Liao, J. Song, B. Li, G. Wu, J. Magn. Magn. Mater. 378, 232 (2015).

[8] M. Huang, Ch. Wu, Y. Jiang, M. Yan, J. Alloys Compd. 644, 124 (2015).

[9] P. Kollár, P. Slovenský, D. Olekšáková, M. Jakubčin, Z. Birčáková, J. Füzer, R. Bureš, M. Fáberová, submitted to Scr. Mater..

[10] S. Dobák, J. Füzer, P. Kollár, M. Fáberová, R. Bureš, J. Magn. Magn. Mater. 426, 320 (2017).

[11] A. Nouri, A. Sola, Met. Powder Rep. 73, 276 (2018).

[12] A.H. Taghvaei, H. Shokrollahi, M. Ghaffari, K. Janghorban, J. Phys. Chem. Solids 71, 7 (2010). 